\title{
X-Ray Magnetic Circular Dichroism and Compton Scattering: Application to $5 f$ Electron Systems
}

\author{
N. Kernavanois, J.P. Sanchez*, P. Dalmas de RÉotier \\ AND A. YAOUANC
}

Département de Recherche Fondamentale sur la Matière Condensée SPSMS, CEA-Grenoble, 17 rue des Martyrs, 38054 Grenoble cedex 9, France

\begin{abstract}
The basic properties of magnetism depend strongly on the spin and orbital components of magnetization. Information about the magnetic moments can be gained using new techniques, like X-ray magnetic circular dichroism or Compton scattering, developed at third-generation synchrotron sources. After a brief introduction to the basic principles of these new magnetic tools, examples of experiments on $5 f$-electron based systems are presented.
\end{abstract}

PACS numbers: $87.64 . \mathrm{Ni}, 32.80 . \mathrm{Cy}, 75.25 .+\mathrm{z}, 75.75 .+\mathrm{a}$

\section{Introduction}

Up to the early nineties, neutron scattering and hyperfine interaction measurements (NMR, Mössbauer, etc.) were traditional methods used to explore the microscopic nature of the magnetism of new materials. The experimental situation gained a dramatic impulse with the advent of third-generation synchrotron sources which provide intense photon fluxes with tunable polarization and energy. This led to a profusion of new techniques like X-ray magnetic diffraction (resonant and non-resonant), circular dichroism, Compton scattering, or nuclear forward scattering which have opened up new possibilities to probe magnetism [1-3].

In this paper, we will restrict ourselves to X-ray magnetic circular dichroism (XMCD) and magnetic Compton scattering (MCS) techniques which both require circularly polarized X-rays and the presence of a net magnetization in the studied sample (generally a ferro- or a ferrimagnet). In the case of XMCD the photon

* corresponding author 
energy is tuned around the threshold (absorption edge) excitation of a core electron into the unfilled part of the valence band. The integrated intensities of the dichroic signals allow, with the help of sum rules, to determine the individual orbital and spin moments of a given electronic shell of a specific element $[4,5]$. MCS is a technique solely sensitive to spin magnetization and, because it is an incoherent scattering process, only the total spin magnetization is measurable [6, 7]. However, in some favourable cases site or even shell specific information can be extracted from the analysis of the magnetic Compton profile.

To illustrate the possibilities of both techniques we will present examples of experiments done recently on uranium compounds [8-18]. Driving these series of experiments was theory which made interesting predictions about the orbital moments of itinerant $f$ electrons [19]. It was confirmed experimentally that $f$ electrons lose some of their orbital character in itinerant compounds. The second interesting aspect was the demonstration that $d$-conduction electrons could carry weak orbital moment [15]. Finally, we will show that XMCD experiments on uranium based multilayers [15] provide unique information difficult to attain by other methods like neutron reflectometry [20].

\section{Magnetism - spin and orbital moments}

Orbital and spin magnetic moments are the most fundamental quantities for understanding the macroscopic magnetic properties of matter. These moments are determined by the interplay among several effects: hybridization (or band width $W)$, exchange $\left(\Delta_{\text {exch }}\right)$, and Coulomb $\left(\Delta_{\mathrm{C}}\right)$ interactions, spin-orbit coupling $\left(\Delta_{\mathrm{SO}}\right)$, and crystal fields $\left(\Delta_{\mathrm{CF}}\right)$. Typical energy scales for these different interactions for actinides (5f), lanthanides $(4 f)$ and $3 d$ transition metals are summarized in Table I.

\section{TABLE I}

Typical energy scale (in eV) for bandwidth $(W)$, exchange $\left(\Delta_{\text {exch }}\right)$, Coulomb $\left(\Delta_{\mathrm{C}}\right)$, spin-orbit $\left(\Delta_{\mathrm{SO}}\right)$ and crystal field $\left(\Delta_{\mathrm{CF}}\right)$ interactions for $5 f, 4 f$, and $3 d$ transition metals.

\begin{tabular}{l|c|c|c}
\hline \hline & $5 f$ & $4 f$ & $3 d$ \\
\hline$W$ & $2-5$ & $1-2$ & $5-10$ \\
$\Delta_{\text {exch }}$ & $\approx 0.01$ & $0.001-0.01$ & $\approx 1$ \\
$\Delta_{\mathrm{C}}$ & $\approx 10$ & $\approx 20$ & $\approx 10$ \\
$\Delta_{\mathrm{SO}}$ & $\approx 0.3$ & $\approx 0.1$ & $\approx 0.05$ \\
$\Delta_{\mathrm{CF}}$ & $0.01-0.1$ & $<0.01$ & $\approx 1$
\end{tabular}


The standard model for $4 f$ magnetism (except for anomalous $4 f$ elements like $\mathrm{Ce}$ ) is based upon the approximation that the $4 f$ states are atomic-like, i.e., the magnetism is localized. The ratio $R=\mu_{L} / \mu_{S}$, where $\mu_{L}$ is the orbital moment and $\mu_{S}$ the spin moment, is easy to calculate. It is given by the expression

$$
\mu_{L} / \mu_{S}=\frac{2-g_{J}}{2\left(g_{J}-1\right)}
$$

where $g_{J}$ is the Lande factor in the Russell-Saunders (RS) coupling.

As long as $\Delta_{\mathrm{SO}} \gg \Delta_{\mathrm{CF}}$, there is no mixing of $J$ states and the value of $R$ is independent of the crystal field ground state. However, the total $4 f$ moment is generally reduced compared to the free-ion $g_{J} J \mu_{\mathrm{B}}$ value.

In contrast, in $3 d$ metals the $3 d$ band widths are large (a few eV), the $3 d$ states are components of the conduction electron band structures and their magnetism is accounted for by itinerant models. The orbital moment is almost completely quenched (e.g. $\mu_{L}=0.14 \mu_{\mathrm{B}}$ and $\mu_{S}=1.64 \mu_{\mathrm{B}}$ for Co). The observed tiny orbital moment arises from the spin-orbit interaction.

As shown in Table I, the $5 f$ band widths of the actinides are in between those of $3 d$ transition metals and lanthanides whereas the spin-orbit interaction $\Delta_{\mathrm{SO}}(5 f)$ is large in comparison. The standard models developed for $4 f$ and $3 d$ metals cannot be, generally, applied to the interpretation of the magnetism of the actinides. In fact, it was shown both theoretically and experimentally that the orbital moments are reduced when the $5 f$ states are itinerant but, unlike the $3 d$ transition metal systems where $\mu_{L} \approx 0$, the itinerant $5 f$ states retain a large orbital character (in many cases the orbital moments overcome the spin contributions). The reduction of the ratio $R$, given by expression (1) where $g_{J}$ is now calculated within the framework of intermediate coupling (IC), compared to the free ion values, reflects somewhat the $5 f-s p d$ hybridization strength [21]. Strongly depressed $R$ values were found in the itinerant systems $\mathrm{UFe}_{2}, \mathrm{UNi}_{2}$, $\mathrm{NpCo}_{2}$, and $\mathrm{PuFe}_{2}[21]$.

Up to recently measurement of the form factor $f(Q)$ by polarized neutron techniques was almost the unique tool which could determine the individual spin and orbital moments [22]. Within the dipolar approximation, the magnetic amplitude is given by

$$
\mu f(Q)=\mu\left[\left\langle j_{0}\right\rangle+C_{2}\left\langle j_{2}\right\rangle\right]
$$

where $C_{2}=\mu_{L} / \mu ;\left\langle j_{0}\right\rangle$ and $\left\langle j_{2}\right\rangle$ are tabulated for given ionic configurations as a function of the modulus of the scattering vector $Q=\sin \theta / \lambda$.

This method is specially well suited for cases where the $5 f$ (or $4 f$ ) orbital and spin contributions are in opposite directions (light $4 f$ and $5 f$ elements) and of comparable magnitude (e.g. $\mathrm{UFe}_{2}$ ). One of the weaknesses of neutron form factor measurements is that they fail to detect $6 d$ (or $5 d$ )-like magnetization densities. Such magnetization densities could be picked up by XMCD or MCS techniques which therefore are complementary to polarized neutron experiments. 


\section{X-ray magnetic circular dichroism}

In an XMCD experiment one measures the difference in absorption $\left(\sigma^{+}-\sigma^{-}\right)$ between left (positive helicity) and right (negative helicity) handed circularly polarized X-rays during the process of electronic transitions from core states to unoccupied valence states (Fig. 1). XMCD requires the presence of a net magnetic moment. It is thus generally restricted to ferromagnets (or ferrimagnets), but it can be applied to paramagnets or even antiferromagnets if they can be polarized by an intense external field. Experimentally the dichroic signal $\left(\sigma^{+}-\sigma^{-}\right)$is observed in the vicinity of an absorption edge. It is only a few percents of the total absorption. It was demonstrated both theoretically and experimentally that inverting the external field (often easier) or the sign of the circular polarization gives the same dichroic spectrum. The choice of the absorption edge makes XMCD a unique element and electronic shell specific tool.

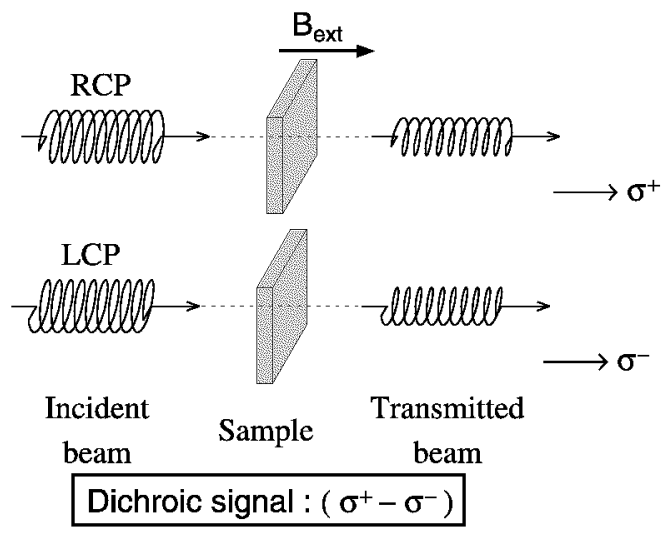

Fig. 1. Normal geometry for XMCD experiments. The XMCD signal is the difference in the absorption when either the handedness of the X-ray (RCP/LCP) or the direction of the external field $\left(B_{\text {ext }}\right)$ is reversed.

The origin of the XMCD signal is easiest to understand by taking an atomic model as a starting point and considering the electronic transitions governed by the electric dipole $\left(E_{1}\right)$ selection rules: $\Delta l= \pm 1$ and $\Delta m=+1$ (positive helicity, left polarization), and $\Delta m=-1$ (negative helicity, right polarization) where $\Delta l, \Delta m$ are the difference between the initial and final orbital and magnetic quantum numbers, respectively. Figure 2 illustrates how the selection rules lead to the observation of a dichroic signal. For photons with positive helicity the transition from the core state $|0,0\rangle$ to the unoccupied $|1,1\rangle$ state is allowed. In contrast, for photons with negative helicity the transition to the final state is forbidden as the $|1,-1\rangle$ state is already occupied. Hence, a large XMCD signal results.

For rare-earth compounds the $M_{4,5}$ and $L_{2,3}$ edges are the most studied [24] whereas for $3 d$ transition metal compounds the $L_{2,3}$ edges are the most use- 


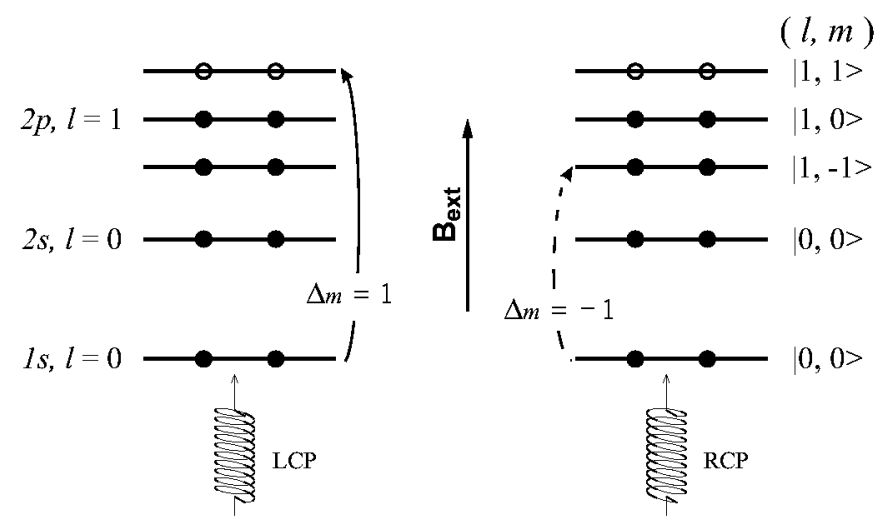

Fig. 2. Simplified energy level diagram of an atom containing eight electrons and illustration how the electric dipole selection rules lead to a large XMCD signal (after Als-Nielsen and Mc Morrow [23]).

ful [25, 26]. XMCD experiments on actinide compounds were so far essentially limited to the $M_{4,5}$ edges (Table II). All these edges are split by the spin-orbit coupling acting on the core-hole. They allow to probe the $f$ ( $M_{4,5}$ edges) or $d\left(L_{2,3}\right.$ edges) shells which carry a magnetic moment.

TABLE II

Useful absorption edges for XMCD studies of $3 d$ transition metal, rare-earth and actinide compounds.

\begin{tabular}{c|c|c}
\hline \hline $\begin{array}{c}3 d \text { transition metal } \\
\text { compounds }\end{array}$ & $2 p_{1 / 2,3 / 2} \rightarrow 3 d$ & $L_{2,3}(400-1000 \mathrm{eV})$ \\
\hline Rare-earth compounds & $3 d_{3 / 2,5 / 2} \rightarrow 4 f$ & $M_{4,5}(800-1600 \mathrm{eV})$ \\
& $2 p_{1 / 2,3 / 2} \rightarrow 5 d$ & $L_{2,3}(5-10 \mathrm{keV})$ \\
\hline Actinide compounds & $3 d_{3 / 2,5 / 2} \rightarrow 5 f$ & $M_{4,5}(3-5 \mathrm{keV})$
\end{tabular}

Two important magneto-optical sum rules have been derived by Thole et al. [4] and Carra et al. [5] which allow to deduce element specific orbital $\left(-\left\langle L_{z}\right\rangle \mu_{\mathrm{B}}\right)$ and spin $\left(-2\left\langle S_{z}\right\rangle \mu_{\mathrm{B}}\right)$ moments from XMCD experiments.

The first (or orbital) sum rule links the integrated dichroic signal over the two edges to the ground state expectation value of the $z$-component of the angular momentum $\boldsymbol{L}$ acting on the shell which receives the photoelectron in the final state.

For a transition between a core level $c$ towards a valence level $l$ with $n$ electrons in the ground state, the orbital sum rule writes

$$
\frac{\left\langle L_{z}\right\rangle}{n_{\mathrm{h}} l}=\frac{\int_{J++J^{-}}\left(\sigma^{+}-\sigma^{-}\right) \mathrm{d} \omega}{\int_{J++J^{-}}\left(\sigma^{+}+\sigma^{-}+\sigma_{0}\right) \mathrm{d} \omega} .
$$


$n_{\mathrm{h}}$ is the number of holes in the $l$ shell $\left(n_{\mathrm{h}}=4 l+2-n\right) ;\left(\sigma^{+}+\sigma^{-}+\sigma_{0}\right) / 3$ corresponds to the non-polarized absorption spectrum. The integral over $J^{+}$and $J^{-}$is over the $J=c+1 / 2$ and $J=c-1 / 2$ edges energy range. For $M_{4,5}$ edges, $c=2$ and $l=3$, whereas for $L_{2,3}$ edges, $c=1$ and $l=2$.

The second (or spin) sum rule relates a linear combination of the XMCD signals at the core split edges to the average values of the operators $\left\langle S_{z}\right\rangle$ and $\left\langle T_{z}\right\rangle$ acting on the shell which receives the photoelectron in the final state. Keeping the same notation as above and assuming the energy separation between the two edges is large enough to allow integration over separate energy ranges, the spin sum rule writes

$$
\frac{2}{3 n_{\mathrm{h}}}\left(\left\langle S_{z}\right\rangle+\frac{2 l+3}{l}\left\langle T_{z}\right\rangle\right)=\frac{\int_{J+}\left(\sigma^{+}-\sigma^{-}\right) \mathrm{d} \omega-\frac{l}{l-1} \int_{J^{-}}\left(\sigma^{+}-\sigma^{-}\right) \mathrm{d} \omega}{\int_{J++J^{-}}\left(\sigma^{+}+\sigma^{-}+\sigma_{0}\right) \mathrm{d} \omega} .
$$

$\left\langle S_{z}\right\rangle$ is the ground state average value of the spin operator $S$ and $\left\langle T_{z}\right\rangle$ of the magnetic dipolar operator $\left(\boldsymbol{T}=\sum_{i} \boldsymbol{s}_{i}-3 \boldsymbol{r}_{i}\left(\boldsymbol{r}_{i} \cdot \boldsymbol{s}_{i}\right) / \boldsymbol{r}_{i}^{2}\right) .\left\langle T_{z}\right\rangle$ is related to the anisotropy of the local magnetic field produced by the spin when the valence cloud is distorted either by spin-orbit or crystal field interactions. It is often difficult to separate $\left\langle T_{z}\right\rangle$ and $\left\langle S_{z}\right\rangle$ although it has been suggested that $\left\langle T_{z}\right\rangle$ for $3 d$ metals is negligible with respect to $\left\langle S_{z}\right\rangle$. This is generally not the case for $4 f$ and $5 f$ elements where $\left\langle T_{z}\right\rangle$ and $\left\langle S_{z}\right\rangle$ can be of comparable magnitude. The values of $\left\langle L_{z}\right\rangle,\left\langle S_{z}\right\rangle$, and $\left\langle T_{z}\right\rangle$ computed for $\mathrm{U}^{3+}\left(5 f^{3}\right)$ and $\mathrm{U}^{4+}\left(5 f^{2}\right)$ free ions are given in Table III.

\section{TABLE III}

Calculated values of $\left\langle L_{z}\right\rangle,\left\langle S_{z}\right\rangle,\left\langle T_{z}\right\rangle$, ratio $R$ between orbital and spin moments, and total $5 f$ magnetic moment $\mu$ for the uranium $5 f^{n}$ atomic ground state configurations within the framework of intermediate coupling [27].

\begin{tabular}{c|c|c|c|c|c}
\hline \hline Configuration & $\left\langle L_{z}\right\rangle$ & $\left\langle S_{z}\right\rangle$ & $\left\langle T_{z}\right\rangle$ & $R$ & $\mu\left[\mu_{\mathrm{B}}\right]$ \\
\hline $\mathrm{U}^{4+}: 5 f^{2}$ & -4.70 & 0.70 & 0.81 & -3.36 & 3.30 \\
$\mathrm{U}^{3+}: 5 f^{3}$ & -5.57 & 1.07 & 0.66 & -2.60 & 3.43
\end{tabular}

The sum rules have been applied with success to the $M_{4,5}$ edges of rare-earth and actinides [24] and to the $L_{2,3}$ edges of $3 d$ transition metals [26]. However, it was shown that they fail at the $L_{2,3}$ edges of rare-earth compounds with $4 f$ moments [24]. Applications of the sum rules require a thorough analysis of the different approximations under which they are valid [28].

\section{X-ray magnetic Compton scattering}

Compton scattering is an incoherent process which involves inelastic scattering of photons by electrons (Fig. 3). The scattering for polarized photons from a 
stationary free electron is described by the cross-section written as [29]:

$$
\begin{aligned}
\frac{\mathrm{d} \sigma}{\mathrm{d} \Omega}= & \frac{r_{0}^{2}}{2}\left(\frac{k_{2}}{k_{1}}\right)^{2}\left[1+\cos ^{2} \theta+P_{l} \sin ^{2} \theta+\frac{\hbar\left(k_{1}-k_{2}\right)}{m c}(1-\cos \theta)\right. \\
& \left.+(\cos \theta-1) P_{c} \tau \frac{\hbar\left(k_{1} \cos \theta+k_{2}\right)}{m c}\right] .
\end{aligned}
$$

$\boldsymbol{k}_{1}\left(\boldsymbol{k}_{2}\right)$ are the wave vectors of the incident (scattered) photons, $\theta$ is the scattering angle, $r_{0}$ - the Thomson scattering length $\left(2.818 \times 10^{-15} \mathrm{~m}\right), P_{l}$ and $P_{\mathrm{c}}$ are the degrees of linear and circular polarization of the beam, $m$ is the electron rest mass, $c$ - the velocity of light and $\tau$ is a unit vector indicating the electron spin direction. There is no term associated with the orbital magnetization because within the impulse approximation the interaction time is much smaller than the orbital period $[6,7]$.
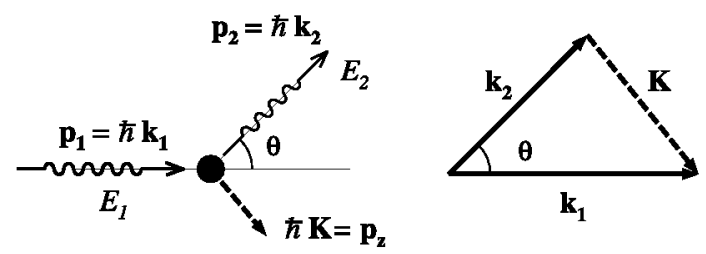

Fig. 3. Schematic illustration of Compton scattering. A photon with energy $E_{1}$ and momentum $\hbar \boldsymbol{k}_{1}$ scatters from an electron at rest with energy $m c^{2}$. The electron recoils with a momentum $\boldsymbol{p}_{z}=\hbar \boldsymbol{K}=\hbar\left(\boldsymbol{k}_{1}-\boldsymbol{k}_{2}\right)$ whereas the scattered photon possesses an energy $E_{2}$ and a momentum $\hbar \boldsymbol{k}_{2}$.

In practice, the electrons will always possess linear momentum and for a fixed scattering angle, the energy spread of the scattered beam due to the motion of the scatterer yields the quantity called the Compton profile denoted $J\left(p_{z}\right)$ (Fig. 4).

It is the probability of finding an electron in the scatterer with a component of momentum $p_{z}$ along the scattering vector $\boldsymbol{K}$. It is defined as the projection $n(\boldsymbol{p})$ of the electron momentum density as follows:

$$
J\left(p_{z}\right)=\iint n(\boldsymbol{p}) \mathrm{d} p_{x} \mathrm{~d} p_{y} .
$$

The magnetic Compton profile relies on the spin resolved quantity. It is given by

$$
J_{m}\left(p_{z}\right)=\iint\left[n^{\uparrow}(\boldsymbol{p})-n^{\downarrow}(\boldsymbol{p})\right] \mathrm{d} p_{x} \mathrm{~d} p_{y}
$$

where $n^{\dagger}(p)$ and $n^{\downarrow}(p)$ are spin up and spin down distributions.

Actually, the cross-section for the Compton scattering consists of a spin-independent term called the charge term, plus a term which is linearly dependent 


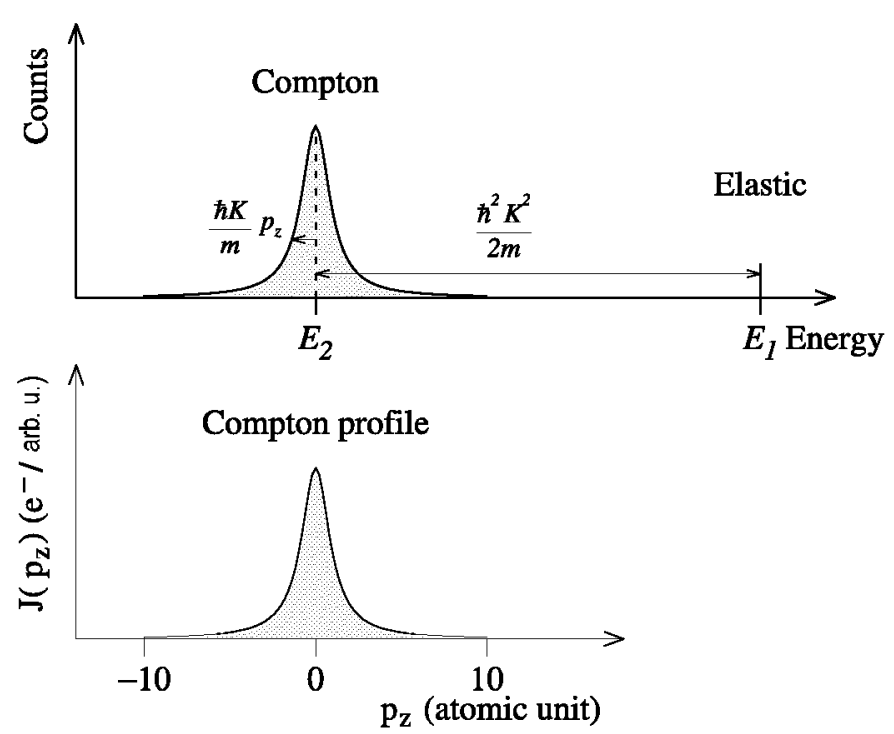

Fig. 4. Compton scattering process when the scatterer has a finite momentum. The spread in energy of the Compton peak (Doppler shift) due to the motion of the electrons in the scatterer yields the electronic momentum distribution called the Compton profile $J\left(p_{z}\right)$.

on the initial electron spin, the magnetic term [1]. This is best illustrated by examining the differential cross-section $\frac{\mathrm{d}^{2} \sigma}{\mathrm{d} \Omega \mathrm{d} E_{2}}$, which follows from expression $(5)$ :

$$
\frac{\mathrm{d}^{2} \sigma}{\mathrm{d} \Omega \mathrm{d} E_{2}}=\frac{m}{\hbar K}\left[\left(\frac{\mathrm{d} \sigma}{\mathrm{d} \Omega}\right)_{c} J_{e}\left(p_{z}\right)+\left(\frac{\mathrm{d} \sigma}{\mathrm{d} \Omega}\right)_{m} J_{m}\left(p_{z}\right)\right]
$$

with

$$
\left(\frac{\mathrm{d} \sigma}{\mathrm{d} \Omega}\right)_{c}=\frac{r_{0}^{2}}{2}\left(\frac{k_{2}}{k_{1}}\right)^{2}\left[1+\cos ^{2} \theta+P_{l} \sin ^{2} \theta+\frac{h\left(k_{1}-k_{2}\right)}{m c}(1-\cos \theta)\right]
$$

and

$$
\left(\frac{\mathrm{d} \sigma}{\mathrm{d} \Omega}\right)_{m}=\frac{r_{0}^{2}}{2}\left(\frac{k_{2}}{k_{1}}\right)^{2}\left[(\cos \theta-1) P_{c} \boldsymbol{\tau} \frac{\hbar\left(\boldsymbol{k}_{1} \cos \theta+\boldsymbol{k}_{2}\right)}{m c}\right] .
$$

Inspection of these equations shows that the magnetic contribution, i.e, the magnetic Compton profile can be isolated from the difference in cross-sections when the direction of the spin vector $\boldsymbol{\tau}$ is reversed by applying an external magnetic field. Moreover, Eq. (10) indicates that the magnetic signal is maximized by working at high energies and at backscattering geometry $\left(\theta \approx 180^{\circ}\right)$. The magnetic scattering is only a small fraction (generally smaller than 1\%) of the total scattering.

The area under the Compton profile, $J\left(p_{z}\right)$ integrated over all momenta, is equal to the total number of electrons $Z$ involved in the scattering process, 
$\int J\left(p_{z}\right) \mathrm{d} p_{z}=Z$, whereas the area under the magnetic Compton profile is equal to the spin moment of the investigated sample, $\int J_{m}\left(p_{z}\right) \mathrm{d} p_{z}=\mu_{S}$. The measurements are generally calibrated by measuring, with the same experimental setup and geometry, the magnetic Compton profile of ferromagnetic iron which has a well-known spin moment $\left(\mu_{S}^{\mathrm{Fe}}=2.12 \mu_{\mathrm{B}}\right)$.

The measured magnetic Compton profile of a ferro- or ferrimagnetic sample contains contributions from all electrons with unpaired spins. However, the site (or even shell) spin moments can be deduced because the momentum distributions associated with each site (shell) are generally significantly different. As an example we show in Fig. 5 the atomic Compton profile of Rh $4 d$, Ce $4 f$, and Ce $5 d$ used to analyse the magnetic Compton profile of the ferromagnet $\mathrm{CeRh}_{3} \mathrm{~B}_{2}$ [31]. One can notice that the Ce $4 f$ electrons are more tightly bound and therefore have a broader Compton profile than the Ce $5 d$ electrons which are diffuse and have a less extended profile.

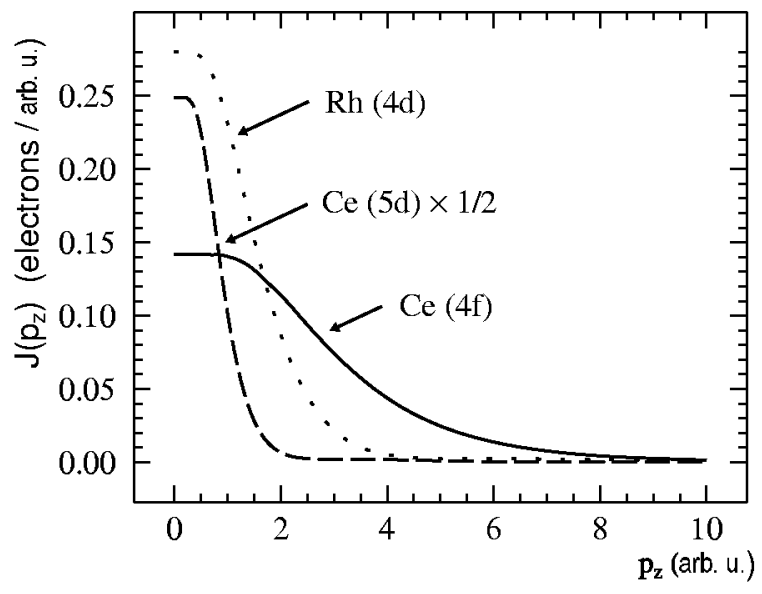

Fig. 5. Atomic Compton profiles of Rh $4 d$, Ce $5 d$ and Ce $4 f$ (data taken from Ref. [30]). Their different profiles allowed to evaluate the contributions of these electronic shells to the spin moment of $\mathrm{CeRh}_{3} \mathrm{~B}_{2}$ [31].

In summary, from the analysis of a magnetic Compton profile one can extract: (i) the sign (i.e. parallel or antiparallel to the magnetization) and magnitude of the spin moment of the investigated ferro- or ferrimagnetic sample, (ii) possibly one can distinguish the contribution of different electronic shells from different chemical elements [32].

\section{Application of XMCD and MCS to $5 f$-electron systems}

In this section we will first report on XMCD and/or MCS experiments performed on bulk uranium compounds with different degree of hybridization: $\mathrm{USb}_{0.5} \mathrm{Te}_{0.5}$ (localized) [10], US (weakly hybridized) [8, 15], and $\mathrm{UFe}_{2}$ (strongly 
hybridized) [9]. Then, we will present recent XMCD results obtained on a series of multilayers made with uranium.

$$
\text { 5.1. US } b_{0.5} T e_{0.5}, U S \text {, and } U F e_{2}
$$

$\mathrm{USb}_{0.5} \mathrm{Te}_{0.5}$ and US which crystallize both in the rock-salt ( $\mathrm{NaCl}$ ) type structure are very anisotropic ferromagnets $\left(T_{\mathrm{C}} \approx 200 \mathrm{~K}\right.$ and $180 \mathrm{~K}$, respectively) with the easy axis along the [111] direction. The saturation magnetic moment of the uranium atom has a value of $\approx 2.6 \mu_{\mathrm{B}}$ for $\mathrm{USb}_{0.5} \mathrm{Te}_{0.5}$ and $1.7 \mu_{\mathrm{B}}$ for US. USb $0.5 \mathrm{Te}_{0.5}$ is considered as a localized system with a purely ionic $\mathrm{U}^{3+}$ configuration $\left(5 f^{3}\right)$. In contrast, US is classified as an itinerant magnet; the $5 f$ electron count is estimated, from band structure calculations, to amount to 2.54. Moreover, it was anticipated, by comparing neutron and bulk magnetization data, that the $6 d$ electrons in US are polarized antiparallel to the $5 f$ moment [33].

$\mathrm{UFe}_{2}$ crystallizes in the cubic Laves phase (C15 structure) and is ferromagnetic below $165 \mathrm{~K}$. Evidence for strong hybridization between the $\mathrm{U} 5 f$ and Fe $3 d$ electrons was first provided by theory and confirmed by neutron form factor measurements [34]. The moment on the $\mathrm{U}$ atom is almost zero because of the cancellation of the orbital and spin moments, which are both about $0.23 \mu_{\mathrm{B}}$, but are oppositely directed. The hybridization also modifies the $\mathrm{Fe}$ magnetic moment which is substantially reduced from $2.2 \mu_{\mathrm{B}}$ in pure Fe to $0.6 \mu_{\mathrm{B}}$ [34].

The XMCD experiments at the uranium $M_{4,5}$ edges were carried out at the European Synchrotron Radiation Facility (ESRF) on the beamline ID12A using the total fluorescence yield detection method. The absorption and the dichroic spectra obtained after correction of the raw data for self-absorption effects and energy dependence of the circular polarization rate are presented in Fig. 6 for US [15]. Similar spectra were found for $\mathrm{USb}_{0.5} \mathrm{Te}_{0.5}[10]$ whereas the dichroic signals are about 15 times smaller for $\mathrm{UFe}_{2}$ [9]. This reflects that the orbital and spin
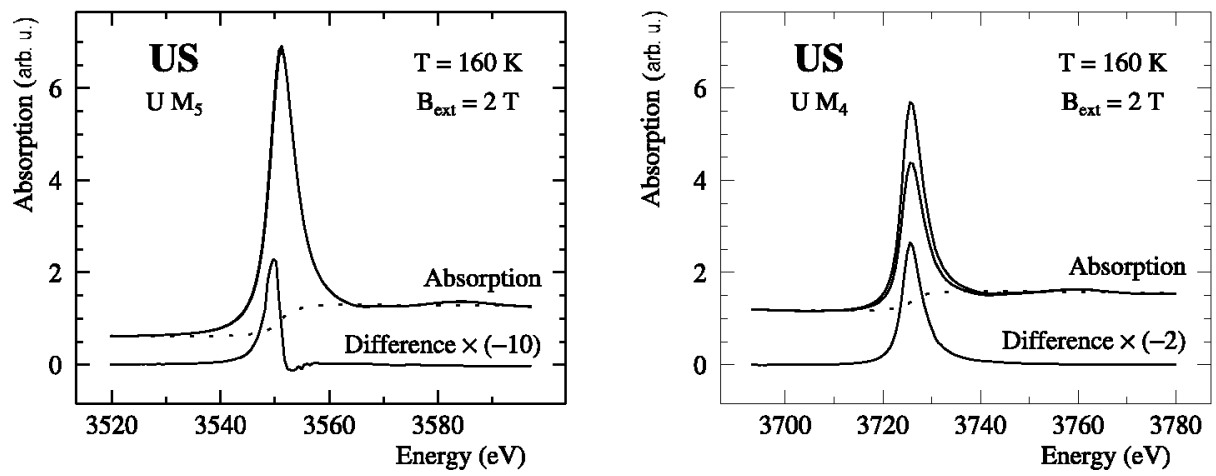

Fig. 6. Absorption and dichroic spectra at the $M_{4,5}$ edges of uranium in US at $160 \mathrm{~K}$ and $2 \mathrm{~T}$. For each edge, the two absorption spectra correspond to the two opposite directions of $B_{\text {ext }}$. Note that the difference is not visible at the $M_{5}$ edge. The dotted line is the step-like function used for background determination [15]. 
contributions are about an order of magnitude weaker in the latter compound. A common feature for all three samples is that the dichroic signal at the $M_{5}$ edge presents some structure (a positive lobe and a small negative splinter) while at the $M_{4}$ edge it consists in a broad feature. It is worth mentioning that, at variance with standard $4 f$ compounds, atomic multiplet calculations fail to reproduce the details of the shape of the $M_{5}$ dichroic signal [9]. Calculations including hybridization effects as well as crystal field interaction are clearly needed. However, it was shown qualitatively that the observed $M_{5}$ line shape can be used to access to the distribution of electrons (holes) in the $5 f$ density of states [14]. One also notices from Fig. 6 that the dichroic signals have the same sign (negative) at both edges. With reference to the first sum rule (Eq. (3)) we deduce that the orbital moments $\mu_{L}^{5 f}$ are parallel to the bulk sample magnetizations and large for US and $\mathrm{USb}_{0.5} \mathrm{Te}_{0.5}$.

The application of the orbital sum rule and normalization to saturation at low temperature give $\mu_{L}^{5 f}=2.7(4), 4.2(2)$, and $0.21(2) \mu_{\mathrm{B}}$ for US, USb $0.5 \mathrm{Te}_{0.5}$, and $\mathrm{UFe}_{2}$, respectively. Note that the uncertainty on $n_{\mathrm{h}}$ in $\mathrm{UFe}_{2}$ (between 11 and 12) was taken into account in the error bar on $\mu_{L}^{5 f}$. To extract $\mu_{S}^{5 f}$ from the XMCD data, we need information about $\left\langle T_{z}\right\rangle$. For $\mathbf{U S b}_{0.5} \mathrm{Te}_{0.5}$, a localized system, we can use the ratio $\left\langle T_{z}\right\rangle /\left\langle S_{z}\right\rangle=0.62$ valid for $\mathrm{U}^{3+}$ free ions (see Table III) and then deduce $\mu_{S}^{5 f}=-1.6(1) \mu_{\mathrm{B}}$ by application of the spin sum rule (Eq. (4)). The ratio $\mu_{L}^{5 f} / \mu_{S}^{5 f} \approx-2.6(3)$ is close to the theoretical $\mathrm{U}^{3+}$ free ion value (see Table III).

Let us now deal with the case of $\mathrm{UFe}_{2}$. The XMCD value of $\mu_{L}^{5 f}$ is in excellent agreement with the result given by polarized neutron scattering [34]. Because $\mathrm{UFe}_{2}$ is a strongly hybridized itinerant ferromagnet it is expected that $\left\langle T_{z}\right\rangle$ is strongly reduced with respect to its atomic value. This is in line with the observed very weak anisotropy. Moreover, band-structure calculations and experiments have shown that, in the bulk, the itinerant magnetic systems $\mathrm{Fe}, \mathrm{Co}, \mathrm{Ni}$ are characterized by $\left\langle T_{z}^{3 d}\right\rangle /\left\langle S_{z}^{3 d}\right\rangle$ lower than 0.01 [26]. If we set $\left\langle T_{z}\right\rangle=0$ we get from (Eq. (4)) $\left\langle S_{z}\right\rangle=0.11(1)$ and $\mu_{S}^{5 f}=-0.22(2) \mu_{\mathrm{B}}$ in accordance with the neutron data. These results were broadly confirmed by MCS experiments which in addition provided information on the orientation and size of the diffuse and iron spin contributions [16].

We will now turn to the more difficult case of US which can neither be considered as localized nor as fully itinerant. Indeed, setting $\left\langle T_{z}\right\rangle$ to zero or the ratio $\left\langle T_{z}\right\rangle /\left\langle S_{z}\right\rangle$ to either $\mathrm{U}^{4+}$ or $\mathrm{U}^{3+}$ free ion values leads to non-physical results. To solve this puzzling problem we performed MCS experiments on US [15] at the end-station of the beamline ID15A at ESRF.

Figure 7 shows the magnetic Compton profile (MCP) of US. It is negative reflecting that the spin moment of US is antiparallel to the external field $B_{\text {ext }}$. This is expected because the magnetism of US arises mainly from the $5 f$ electrons and following Hund's rule $\mu_{S}^{5 f}$ is antiparallel to $\mu_{L}^{5 f}$. The analysis of the MCP provides not only the total spin moment of US, but it allows also to separate the 


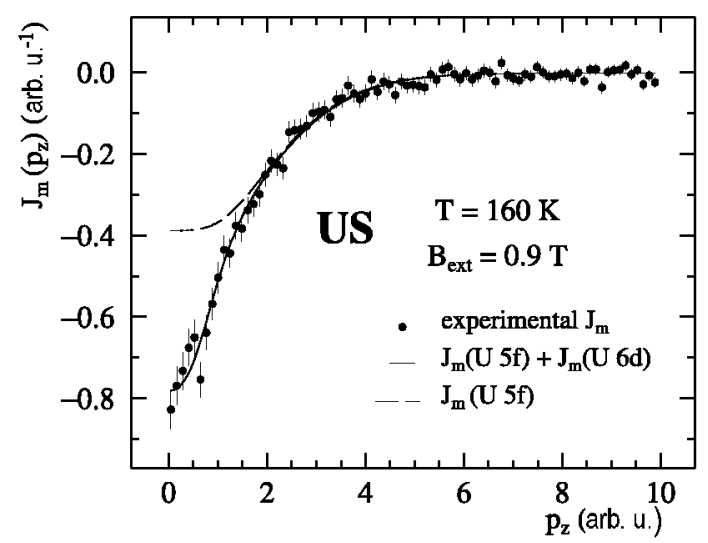

Fig. 7. Magnetic Compton profile of US (filled circles) at $160 \mathrm{~K}$ and $0.9 \mathrm{~T}$. The energy of the incident photons was $104.36 \mathrm{keV}$ and the scattering angle $\theta$ was set to $171.2^{\circ}$. The fit (solid line) was obtained from $5 f$ and $6 d$ free atom calculated profiles of uranium convoluted by the experimental resolution function. The dashed line is the contribution of the $5 f$ electrons of uranium [15].

$5 f$ and $6 d$ spin contributions (Fig. 7). This is because the characteristic $\mathrm{U} 5 f$ and $\mathrm{U} 6 d$ atomic momentum distributions, and hence their projections which constitute the Compton profile, are different. Actually, the momentum density above 2 a.u. arises only from $5 f$ electrons. The combination of spin-resolved Compton data with the total and $5 f$ moments as determined by bulk magnetization and neutron diffraction experiments leads to the determination of the orbital moments carried by the uranium $5 f$ and $6 d$ electrons (Table IV). The most original feature arises from the non-vanishing orbital moment for the $\mathrm{U} 6 d$ electrons. This reflects a relatively strong spin-orbit coupling in the $6 d$ band. In addition, the data taken from Table IV, together with the dichroism results which provide a precise estimate $(-1.56(6))$ for the ratio $\left\langle L_{z}\right\rangle /\left(\left\langle S_{z}\right\rangle+3\left\langle T_{z}\right\rangle\right)$, allow to deduce a value for $\left\langle T_{z}\right\rangle(0.41(4))$ in good agreement with the one $(0.36)$ obtained by Shishidou et al. [35] from band-structure calculations. The $\left\langle T_{z}\right\rangle$ value we find seems to suggest a $5 f^{2}$ configuration (after rescaling the 0.81 free ion value of $\left\langle T_{z}\right\rangle$ by the

\section{TABLE IV}

Saturated magnetic moments in US in $\mu_{\mathrm{B}}$ units deduced from MCS experiments values $\left(\mu_{S}, \mu_{S}^{5 f}, \mu_{S}^{6 d}\right)[15]$ combined with magnetization $(\mu=1.55(2))$ and neutron scattering $\left(\mu^{5 f}=1.70(3)\right)$ data [36].

\begin{tabular}{c|c|c|c|c|c}
\hline \hline$\mu_{S}$ & $\mu_{L}$ & $\mu_{L}^{5 f}$ & $\mu_{S}^{5 f}$ & $\mu_{S}^{6 d}$ & $\mu_{L}^{6 d}$ \\
\hline$-1.33(9)$ & $2.88(9)$ & $2.67(8)$ & $-0.97(7)$ & $-0.36(3)$ & $0.21(5)$
\end{tabular}


factor $1.7 / 3.30$ ) whereas the ratio $R=\mu_{L}^{5 f} / \mu_{S}^{5 f}=-2.75(22)$ lies close to the $5 f^{3}$ configuration value (Table III). We must however be aware that neutron, XMCD, or MCS results have been deduced using free atom parameters.

\subsection{U-As/Co multilayers}

Following the discovery of large magneto-optical Kerr effects (MOKE) in uranium compounds at low temperature [37] much attention was paid to the fabrication of materials containing uranium which exhibit large MOKE at room temperature. After unsuccessful attempts involving U-As films doped with Co [38], the interest turned to U-As/Co multilayers which present large Kerr rotation at room temperature. This observation was ascribed to an exchange induced magnetic moment in the $\mathbf{U}$-As layers by the cobalt [39]. To confirm this claim, polarized neutron reflectivity measurements were undertaken but they fail to determine unambiguously the magnitude of the moments in the constituent layers owing to the large ferromagnetic signal from the cobalt [20]. This led us to perform XMCD measurements at the uranium $M_{4,5}$ edges in order to probe the $5 f$ magnetism and determine the uranium moment.

We examined three of these multilayers with different combinations of amorphous $\mathrm{U}-\mathrm{As}$ and pure crystalline Co written in the form

$$
\mathrm{Co}(200 \AA)\left[\mathrm{U}-\mathrm{As}(t \AA) / \mathrm{Co}(20 \AA]_{n}\right.
$$

with U/As ratio of $\approx 1.5$ and $t=40 \AA, n=20 ; t=60 \AA, n=15 ; t=80 \AA$, $n=12$, respectively. They were fabricated at IBM and their properties studied using techniques including polar Kerr rotations and SQUID magnetometry [39, 40].

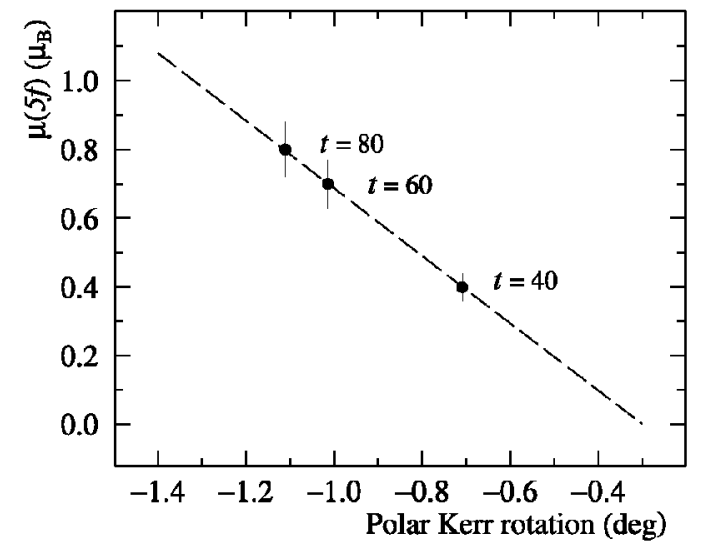

Fig. 8. Uranium moment deduced from the XMCD measurements versus the maximum polar Kerr rotation for the $\mathrm{Co}(200 \AA)[\mathrm{U}-\mathrm{As}(t \AA) / \mathrm{Co}(20 \AA)]_{n}$ multilayers with $t=80$, 60 , and $40 \AA$. The XMCD experiments have been performed at $35 \mathrm{~K}$ and $4 \mathrm{~T}$ while the MOKE values correspond to $10 \mathrm{~K}$ and $3 \mathrm{~T}$ [39]. 
From a first set of XMCD experiments at $35 \mathrm{~K}$ and $B_{\text {ext }}=4 \mathrm{~T}$ it was concluded that the $\mathbf{U}$-As layers carry a uranium magnetic moment in all investigated multilayers. Application of the sum rules assuming an ionic $\mathrm{U}^{3+}$ configuration led to the following estimates for the average uranium moments: $\mu^{5 f}=0.8(2) \mu_{\mathrm{B}}$ $(t=80 \AA), 0.7(2) \mu_{\mathrm{B}}(t=60 \AA)$, and $0.4(1) \mu_{\mathrm{B}}(t=40 \AA)$. The reduction of $\mu^{5 f}$ with decreasing $\mathrm{U}-\mathrm{As}$ layer thickness was ascribed to the interdiffusion of Co in U-As layers leading to the formation of magnetic dead layers of about $13(2) \AA$ at each interface. This result agrees well with the analysis of the Kerr rotation data [39]. Figure 8 clearly demonstrates that the maximum Kerr rotation angle is directly related to the magnitude of the uranium moment. Note that when $\mu^{5 f}=0$, the Kerr rotation angle has a finite value, the so-called optical constant effect.

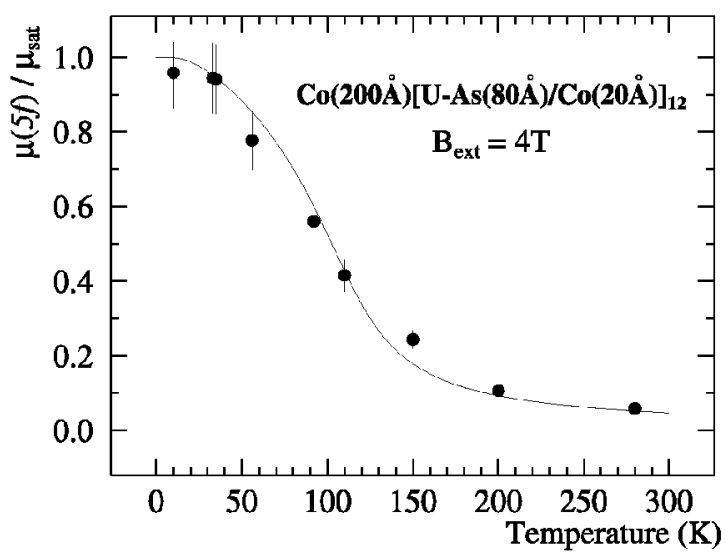

Fig. 9. Temperature dependence of the reduced uranium moment for the multilayer with $t=80 \AA$. The solid line is a calculated curve using a mean field model taking into account that $B_{\text {ext }}=4 \mathrm{~T}$ and assuming that the exchange field due to the Co layers amounts to $B_{\mathrm{exch}}^{\mathrm{Co}} \approx 6 \mathrm{~T}$. The Curie temperature of the U-As layers was set equal to $\approx 100 \mathrm{~K}[15]$.

Among the multilayers investigated only the one with the thicker U-As layers $(t=80 \AA)$ exhibits a dichroic signal at room temperature. The temperature dependence of the reduced uranium moment for the multilayer with $t=80 \AA$ is shown in Fig. 9. The room temperature $U$ moment in the $U$-As layers is about $0.05 \mu_{\mathrm{B}}$, a value significantly smaller than the one $\left(0.14 \mu_{\mathrm{B}}\right)$ estimated by Fumagalli et al. [39] by combining magnetization and Kerr rotation data. The XMCD results provide the most clear evidence for an exchange coupling between the Co and the $\mathrm{U}-\mathrm{As}$ layers at $300 \mathrm{~K}$. There is no increase in the Curie temperature of the $\mathbf{U}-\mathrm{As}$ layers compared to $\mathrm{U}-\mathrm{As} / \mathrm{Co}$ films. 


\section{Conclusions}

XMCD and MCS are now becoming standard techniques for the study of the magnetic properties of materials. Their complementary application with neutron scattering methods has been shown to be crucial in many investigations. XMCD, which needs less material than MCS, has been proven to be an unvaluable method for the study of nanomagnetic samples (films, surfaces, multilayers). Given the interesting results we obtained on multilayers made with uranium, a new field awaits exploration.

One of the most important aspect of these new tools is that they allow to determine the size and direction of the individual orbital and spin moments, and even their anisotropy. The inferred orbital moments carried by the $\mathrm{U} 6 d$ electrons in US or the $\mathrm{Ce} 5 d$ electrons in $\mathrm{CeRh}_{3} \mathrm{~B}_{2}$ [31] came as a surprise because it was generally believed that their band widths are so large that they cannot sustain an orbital moment despite the strong spin-orbit interactions expected for the $\mathrm{U} 6 d$ or Ce $5 d$ bands.

The knowledge of the different orbital and spin contributions to the magnetization density of a material provides the most crucial test not only for band-structure calculations but also for the approximations underlying them. State of the art band calculations allow now even to directly compute either the dichroic signals $[35,41]$ or the magnetic Compton profiles but the latter were so far limited to simple ferromagnets like $\mathrm{Ni}$ or $\mathrm{Gd}[42,43]$.

Despite the rapid progresses made by theory these last years, some experimental results remain puzzling. Among them is the observation of a large enhancement of the dichroic signal when the photons energy is tuned around the $K$-absorption edge $(1 s \rightarrow p)$ of a nominally non-magnetic anion in certain uranium compounds (e.g. US [44]). Although the dichroic signal could involve the hybridization of the anion $p$ orbital with the uranium $5 f$ magnetic states, its giant enhancement (250 times bigger than the signal at the iron $K$ edge in pure ferromagnetic iron) does not imply a large moment at the $S$ atom (an upper bound of the $\mathrm{S}$ moment was estimated by neutron scattering to amount to $0.02 \mu_{\mathrm{B}}$ ) [36].

\section{Acknowledgments}

We are indebted to all our colleagues who contributed to the work described here. Special thanks are due to the ID12A and ID15A teams at ESRF.

\section{References}

[1] S.W. Lovesey, S.P. Collins, X-ray Scattering and Absorption by Magnetic Materials, Oxford University Press, Oxford 1996.

[2] Lecture Notes, Magnetism and Synchrotron Radiation, Mittelwihr School, 1996, Eds. E. Beaurepaire, B. Carrière, J.P. Kappler, Les Editions de Physique, Les Ulis 1997. 
[3] O. Leupold, Acta Phys. Pol. A 100, (2001).

[4] B.T. Thole, P. Carra, F. Sette, G. van der Laan, Phys. Rev. Lett. 68, 1943 (1992).

[5] P. Carra, B.T. Thole, M. Altarelli, X. Wang, Phys. Rev. Lett. 70, 694 (1993).

[6] M.J. Cooper, E. Zukowski, S.P. Collins, D.N. Timms, F. Itoh, H. Sakurai, J. Phys., Condens. Matter 4, L399 (1992).

[7] S.W. Lovesey, J. Phys., Condens. Matter 8, L353 (1996).

[8] S.P. Collins, D. Laundy, C.C. Tang, G. van der Laan, J. Phys., Condens. Matter 7, 9325 (1995).

[9] M. Finazzi, Ph. Sainctavit, A.M. Dias, J.P. Kappler, G. Krill, J.P. Sanchez, P. Dalmas de Réotier, A. Yaouanc, A. Rogalev, J. Goulon, Phys. Rev. B 55, 3010 (1997).

[10] P. Dalmas de Réotier, J.P. Sanchez, A. Yaouanc, M. Finazzi, Ph. Sainctavit, G. Krill, J.P. Kappler, J. Goedkoop, J. Goulon, C. Goulon-Ginet, A. Rogalev, O. Vogt, J. Phys., Condens. Matter 9, 3291 (1997).

[11] P. Dalmas de Réotier, J.P. Sanchez, A. Yaouanc, J. Alloys Comp. 271-273, 414 (1998).

[12] W. Grange, M. Finazzi, J.P. Kappler, A. Delobbe, G. Krill, Ph. Sainctavit, J.P. Sanchez, A. Rogalev, J. Goulon, J. Alloys Comp. 275-277, 583 (1998).

[13] A. Yaouanc, P. Dalmas de Réotier, G. van der Laan, A. Hiess, J. Goulon, C. Neumann, P. Lejay, N. Sato, Phys. Rev. B 58, 8793 (1998).

[14] P. Dalmas de Réotier, A. Yaouanc, G. van der Laan, N. Kernavanois, J.P. Sanchez, J.L. Smith, A. Hiess, A. Huxley, A. Rogalev, Phys. Rev. B 60, 10606 (1999).

[15] N. Kernavanois, Ph.D. thesis, University of Grenoble, Grenoble 2000.

[16] P.K. Lawson, M.J. Cooper, M.A.G. Dixon, D.N. Timms, E. Zukowski, F. Itoh, H. Sakurai, Phys. Rev. B 56, 3239 (1997).

[17] H. Sakurai, H. Hashimoto, A. Ochiai, T. Suzuki, M. Ito, F. Itoh, J. Phys., Condens. Matter 7, L599 (1995)

[18] H. Hashimoto, H. Sakurai, H. Oike, F. Itoh, A. Ochiai, T. Suzuki, J. Phys., Condens. Matter 10, 6333 (1998).

[19] O. Eriksson, M.S.S. Brooks, B. Johansson, R.C. Albers, M. Boring, J. Appl. Phys. 69, 5897 (1991).

[20] D. Mannix, W.G. Stirling, D.G. Bucknall, P.W. Haycock, S.D. Brown, G.H. Lander, T.S. Plaskett, Physica B 234-236, 470 (1997).

[21] G.H. Lander, M.S.S. Brooks, B. Johansson, Phys. Rev. B 43, 13672 (1991).

[22] G.H. Lander, in: Handbook on the Physics and Chemistry of the Rare Earths, Vol. 17, Eds. K.A. Gschneidner, Jr., L. Eyring, G.H. Lander, G.R. Chopin, North-Holland, Amsterdam 1993, p. 635.

[23] J. Als-Nielsen, D. Mc Morrow, Elements of Modern X-ray Physics, Wiley, New York 2001, p. 228.

[24] E. Dartyge, F. Baudelet, C. Giorgetti, S. Odin, J. Alloys Comp. 275-277, 526 (1998).

[25] J. Stöhr, J. Magn. Magn. Mater. 200, 470 (1999).

[26] C.T. Chen, Y.U. Idzerda, H.J. Lin, N.V. Smith, G. Meigs, E. Chaban, G.H. Ho, E. Pellegrin, F. Sette, Phys. Rev. Lett. 75, 152 (1995). 
[27] G. van der Laan, B.T. Thole, Phys. Rev. B 53, 14458 (1996).

[28] M. Altarelli, P. Sainctavit, in: Magnetism and Synchrotron Radiation, Mittelwihr School, 1996, Eds. E. Beaurepaire, B. Carriére, J.P. Kappler, Les Editions de Physique, Les Ulis 1997, p. 65.

[29] F.W. Lipps, H.A. Tolhoeck, Physica 20, 85 (1954); ibid. 20, 385 (1954).

[30] F. Biggs, L.B. Mendelsohn, J.B. Mann, At. Data Nucl. Data Tables 16, 201 (1975).

[31] A. Yaouanc, P. Dalmas de Réotier, J.P. Sanchez, Th. Tschentscher, P. Lejay, Phys. Rev. B 57, R681 (1998).

[32] W.G. Stirling, M.J. Cooper, J. Magn. Magn. Mater. 200, 755 (1999).

[33] J. Rossat-Mignot, P. Burlet, G.H. Lander, in: Handbook on the Physics and Chemistry of the Actinides, Vol. 1, Eds. A.J. Freeman, G.H. Lander, North-Holland, Amsterdam 1984, p. 415.

[34] M. Wulff, G.H. Lander, B. Lebech, A. Delapalme, Phys. Rev. B 39, 4719 (1989).

[35] T. Shishidou, T. Oguchi, T. Jo, Phys. Rev. B 59, 6813 (1999).

[36] F.A. Wedgwood, J. Phys. C 5, 2427 (1972).

[37] W. Reim, J. Schoenes, in: Ferromagnetic Materials, Vol. 5, Eds. K.H.J. Buschow, E.P. Wolfarth, North-Holland, Amsterdam 1990, p. 133.

[38] P. Fumagalli, T.S. Plaskett, T.R. Mc Guire, J. Appl. Phys. 73, 6112 (1993).

[39] P. Fumagalli, T.S. Plaskett, D. Weller, T.R. Mc Guire, R.J. Gambino, Phys. Rev. Lett. 70, 230 (1993).

[40] T.S. Plaskett, P. Fumagalli, T.R. Mc Guire, R.J. Gambino, N.A. Bojarczuk, J. Angilello, IEEE Trans. Magn. 28, 2659 (1992).

[41] J. Kunes, P. Novak, M. Divis, P.M. Oppeneer, Phys. Rev. B 63, 205111 (2001).

[42] M.A.G. Dixon, J.A. Duffy, S. Gardelis, J.E. Mc Carthy, M.J. Cooper, S.B. Dugdale, T. Jarborg, D.N. Timms, J. Phys., Condens. Matter 10, 2759 (1998).

[43] J.A. Duffy, J.E. Mc Carthy, S.B. Dugdale, V. Honkimäki, M.J. Cooper, M.A. Alam, T. Jarlborg, S.B. Palmer, J. Phys., Condens. Matter 10, 10391 (1998).

[44] A. Rogalev, J. Goulon, J.P. Sanchez, N. Kernavanois, in: European Synchrotron Radiation Facility Highlights 1999, p. 57. 